“ (C) 2016 IEEE. Personal use of this material is permitted. Permission from IEEE must be obtained for all other uses, in any current or future media, including

reprinting/republishing this material for advertising or promotional purposes, creating new collective works, for resale or redistribution to servers or lists, or reuse of any copyrighted component of this work in other works." 


\title{
Metamaterial-inspired Configurations to Enhance the Directivity of Electrically Small Antennas
}

\author{
Richard W. Ziolkowski ${ }^{1,2}$ \\ ${ }^{1}$ Department of Electrical and Computer Engineering, University of Arizona, Tucson, AZ, USA, ziolkowski@ece.arizona.edu \\ ${ }^{2}$ School of Computing and Communications, University of Technology, Sydney (UTS), Sydney, Australia
}

\begin{abstract}
It has been demonstrated that metamaterialinspired electrically small antennas (ESAs) can be designed to have high radiation efficiencies and even large bandwidths with non-Foster circuit augmentations. However, being electrically small, it still remains a challenge to obtain directivities over interesting bandwidths which exceed those of simple dipoles, especially with only passive constructs. Different classes of metamaterial-inspired ESAs that have successfully produced higher directivities will be reviewed and new configurations will be introduced and discussed.
\end{abstract}

Index Terms-Artificial magnetic conductor, directivity, electrically small antennas, endfire array, Huygens source

\section{INTRODUCTION}

The desire for yet smaller mobile platforms continues to increase. This trend has generated an intense demand for printed electrically small antennas (ESAs) with superior performance characteristics. Generally, omni-directional patterns are desired and they arise naturally from small, compact electric or magnetic dipole radiators [1]-[3]. The latter occur naturally as ESAs, i.e., when the size of the radiating structure is much smaller than the source wavelength. On the other hand, there are a variety of applications for which it is desirable to have higher directivity and/or a large front-to-back ratio, i.e., to have the radiated power emitted primarily into one hemisphere. Examples include biomedical monitoring and onbody systems; point-to-point communications and wireless power transfer; mitigation of cell-phone specific absorption rate (SAR) issues; and radio frequency identification devices (RFIDs).

There have been many approaches reported to obtain higher directivity from ESA configurations. One was enabled by the development of high impedance ground planes $[4,5]$ and bulk artificial magnetic conductors [6], i.e., metastructures and metamaterials acting as in-phase reflectors. A second is to use two or more ESAs in an end-fire array configuration [7-10]. A third is to introduce additional nearfield resonant parasitic elements (NFRP) designed and tuned specifically for directivity enhancement [11]-[13]. A fourth is to consider an orthogonal combination of electric and magnetic dipole radiators to achieve a Huygens source $[14,15]$. These various approaches will be reviewed; the advantages, disadvantages and peculiarities with them will also be discussed.

\section{ARTIFICIAL MAGNETIC CONDUCTORS}

One of the first metamaterial structures considered for antenna applications was the artificial magnetic conductor (AMC). The basic AMC design goal is to achieve, in practice, the behavior of an ideal magnetic conductor, i.e., in-phase unity reflectivity [4] at least to a normally incident plane wave. Many high impedance surfaces (HISs) have been constructed to produce this desired behavior.

The original HIS structures included the Sievenpiper mushroom [16, 17] and Itoh UC-PBG [18] structured ground planes. Both the mushroom type and the frequency selective surface (FSS) type (e.g., the UC-PBG design) surfaces achieve reflectivity with magnitude near one because of the presence of a ground plane, i.e., the vias of the mushrooms are connected to a ground plane and the FSS elements lie on top of a dielectric backed by a ground plane. By adjusting the geometry and materials in the structure, its phase response can be tuned. The in-phase behavior is then obtained at one specified frequency. Subsequently, additional significant efforts are expended to increase the $\pm 45^{\circ}$ bandwidth by decreasing the slope of the phase response through that frequency point. Furthermore, with the presence of its vias, the mushroom surface also acts as an electromagnetic band gap structure for surface waves.

In contrast, the capacitively loaded loop (CLL) bulk metamaterial has no ground plane [6]. Its unit cell and the measured prototype slab are shown in shown in Fig. 1a and $1 b$, respectively. The bulk AMC achieves its properties simply by the choice and design of the CLL elements, the inclusions in its unit cells. The CLL elements are driven by the magnetic (and electric) fields of the electromagnetic wave incident upon them. Their combined scattering responses determine the reflectivity of this metamaterial slab. The unit cells are resonant at a frequency determined by their effective inductance and capacitance. Working at a frequency on the lower side of this resonance where its losses are negligible, a large positive permeability value is obtained, which in turn yields a large impedance value. Working similarly on its high side, one obtains a mu-negative (MNG) response. Furthermore, if the excitation wave is incident towards the capacitive gap, the AMC behavior is obtained. On the other hand, if it is incident towards its backside, an artificial electric conductor behavior is obtained. Thus this bulk metamaterial AMC shares many of the useful properties of the HISs. 


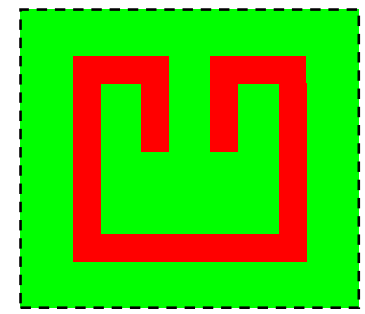

(a) (b)
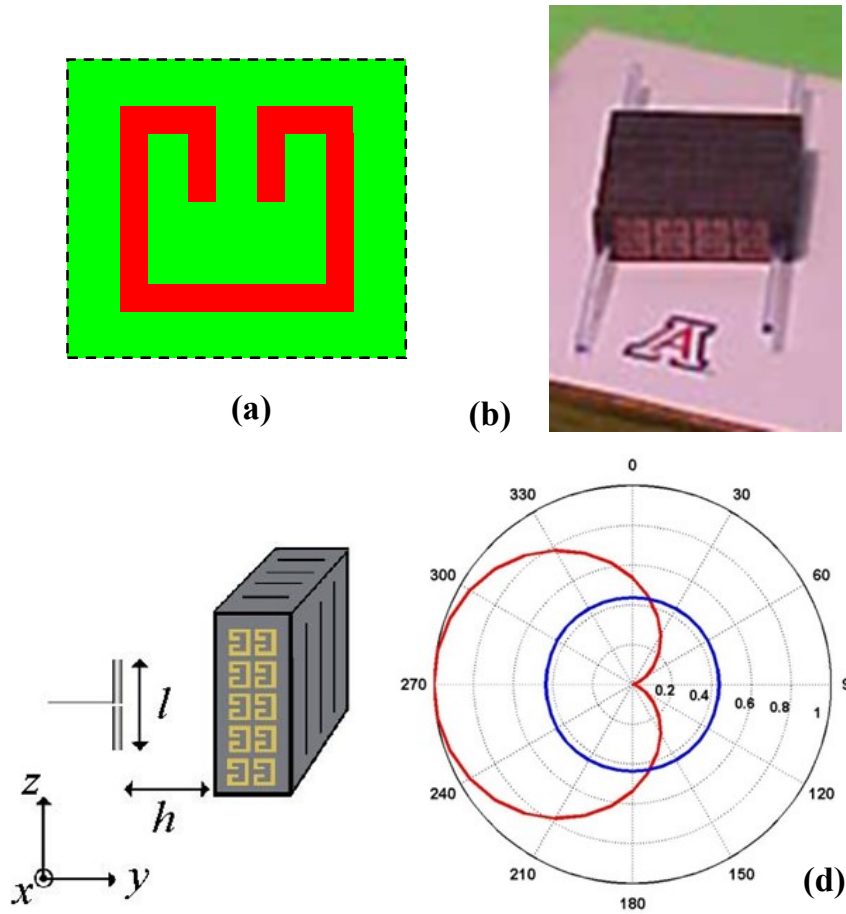

(c)

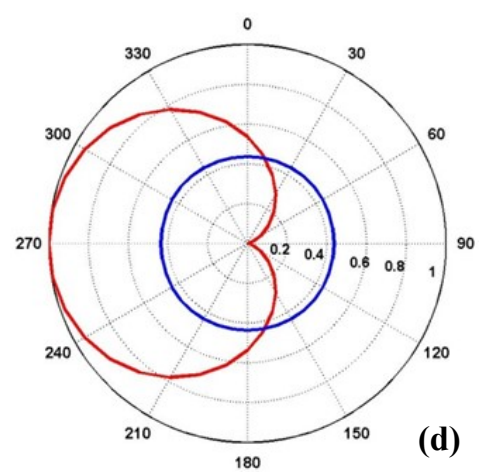

Free-space dipole Dipole antenna / CLL-block

Fig. 1 Capacitively loaded loop (CLL) based artificial magnetic conductor (AMC). (a) CLL unit cell, (b) fabricated AMC block, (c) low profile antenna configuration, and (d) pattern of the excited dipole with and without the AMC block. [6]

Because of its in-phase reflectivity, a dipole radiator parallel to any of these AMC surfaces will have an image that is an in-phase dipole, in contract to the out-of-phase versions obtained with a perfect electric conductor. Thus, in theory, an extremely low-profile antenna is achievable with an AMC if it is thin enough. Moreover, if the surface area of the AMC is large enough, the antenna response is directed only into one half space, as illustrated in Figs. 1c and 1d. In practice, the overall surface behavior is not realized if the radiator is too close to the AMC and only couples to a single element rather than to the entire structure. Furthermore, if the AMC surface is too small, the fields will be radiated into both half spaces.

\section{END-FIRE ARRAYS}

By combining two or more elements (with at least one being driven) together in an end-fire configuration, it has been demonstrated that significant directivity enhancements can be realized [7-10]. An example of this approach is the compact two element array shown in Fig. 2. It is a flexible printed NFRP GPS L1 antenna that is an efficient, directive electrically small radiator [19]. The simple uniplanar design incorporates a capacitively loaded loop (CLL) resonator, which acts as the NFRP element, and a coplanar waveguide (CPW)fed semi-loop antenna. A set of slots are introduced into the CPW feedline ground strips. The resulting meanderline-shaped

CPW ground strips act as a director dipole element that is capacitively coupled to the NFRP element in such a manner that when they are properly tuned, nearly complete matching to the $50 \Omega$ source is achieved with no matching circuit, and the pair acts as a two-element end-fire array. Parameter studies reported in [19] illustrate the nuances of the design and its operating mechanisms. The end-fire realized gain is $3.57 \mathrm{dBi}$ with a $13.44 \mathrm{~dB}$ front-to-back-ratio (FTBR) at its resonance frequency: $1.574 \mathrm{GHz}$ (GPS L1), where the electrical size $\mathrm{ka}=$ 0.97 . The flexibility of the proposed antenna was demonstrated both numerically and experimentally by mounting it on several cylindrical structures whose curvatures vary over a large range and by confirming that there is little impact on its operational frequency, impedance matching, bandwidth and radiation characteristics [19].
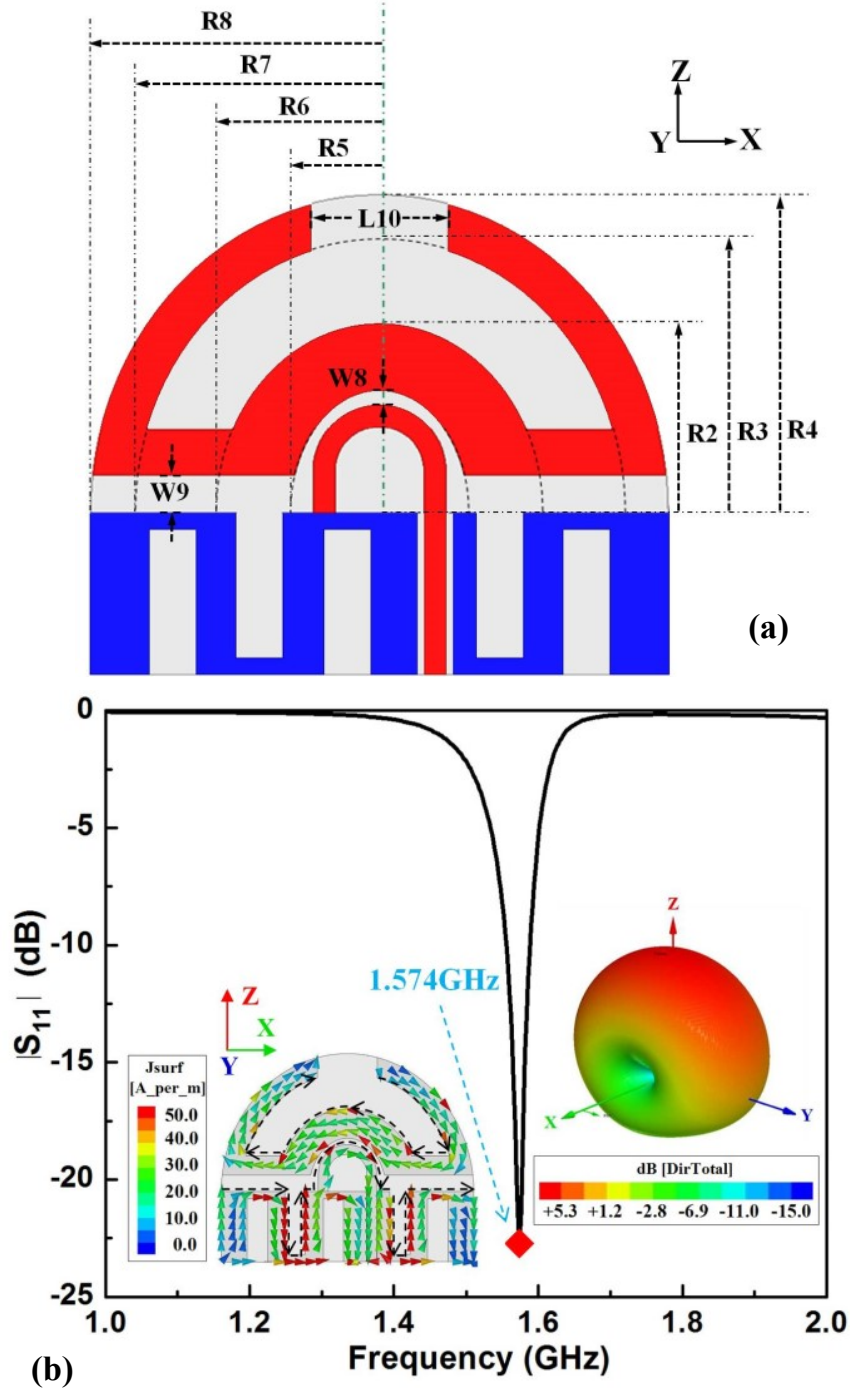

Fig. 2. This printed, flexible, modified CPW-feedline, electrically small antenna operates an end-fire, two element dipole array. Both the modified CPW-feedline and the CLL NFRP element act as electric dipole radiators. (a) Basic design, and (b) $\left|\mathrm{S}_{11}\right|$ values as a function of the excitation frequency. The inset figures represent the current distributions on the metal components and the $3 \mathrm{D}$ directivity pattern. The latter illustrates the enhanced directivity that occurs along the positive $\mathrm{z}$-axis away from the source. [19] 


\section{ACTIVE NFRP ELEMENT}

By introducing more NFRP elements into a metamaterialinspired electrically small antenna system, one obtains more degrees of freedom which can be used to enhance the directivity. These additional NFRP elements can be either passive elements or they can be augmented with a non-Foster (e.g., a negative impedance convertor (NIC)) circuit to increase the bandwidth of the resulting directivity enhancement effects [11]-[13]. This concept is illustrated in Fig. 3.
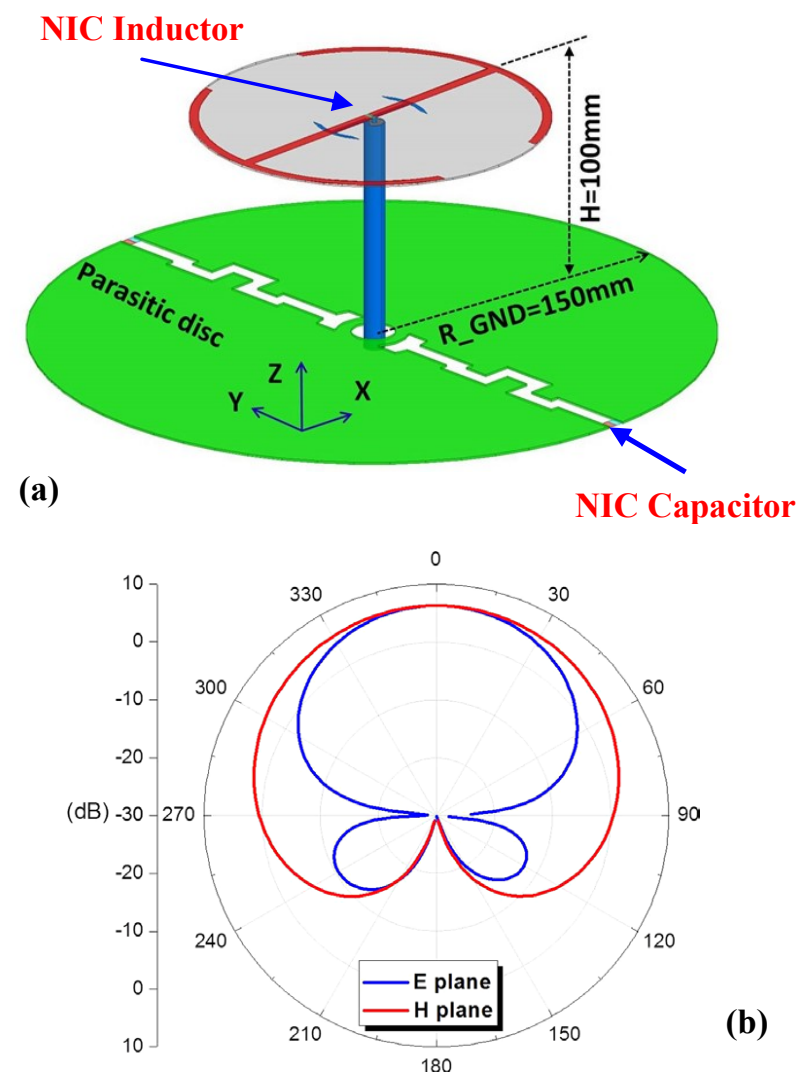

(b)

Fig. 3. Electrically small antenna with non-Foster augmented NFRP elements to achieve high radiation efficiency, large bandwidth, large front-to-back ratio (FTBR) and high directivity. (a) Basic configuration, and (b) the E- and Hplane directivity patterns at $300 \mathrm{MHz}$. [11]

A second NFRP element, the meanderline slot-based conductive disk which was designed and tuned specifically for directivity enhancement, was added to an Egyptian axe dipole (EAD) antenna [20]. The coax-fed driven element is a curved top-hot electric dipole. The EAD NFRP element can be passive or it too can be augmented with a non-Foster circuit, a NIC inductor, to enhance the impedance bandwidth of the resulting EAD antenna [21, 22]. The meanderline-slotted metallic NFRP disk shown in Fig. 3 has two NIC capacitors incorporated into it at the outside ends of the slots. These increase the directivity bandwidth. The overall size of this particular design [11] was ka $=0.94$. It achieved the directivity over quality factor more than 10 times the fundamental bound: $\mathrm{D} / \mathrm{Q}>10 \times(\mathrm{D} / \mathrm{Q})_{\text {bare } \mathrm{EAD}}$. In particular, with a center frequency at $300 \mathrm{MHz}$, it simultaneously achieved high radiation efficiencies $(>81.63 \%)$, high directivities $(>6.25 \mathrm{~dB})$, and large front-to-back-ratios $(>26.71 \mathrm{~dB})$ over a $10.0 \%$ fractional bandwidth.

\section{Huygens SOURCES}

Huygens equivalence principle [3] provides yet another means to achieve higher directivity. By combining together an electric dipole and a magnetic dipole in a plane, one can obtain a Huygens source, i.e., an antenna that radiates primarily into one of the half spaces bounded by that plane. The key design issues are to achieve the same phase center for both radiating elements and to have them both radiate equivalent amounts of power. Additional tuning is necessary to direct the radiated power into the desired half space.

One such design is shown in Fig. 4 [14]. A transmission line-fed printed dipole antenna is the driven radiator. An EAD NFRP element antenna provides the electric dipole response. Two protractor NFRP elements provide the orthogonal magnetic dipole response. By properly tuning the resonances of the electric and magnetic NFRP elements, the directivity pattern shown in Fig. 4 was obtained.

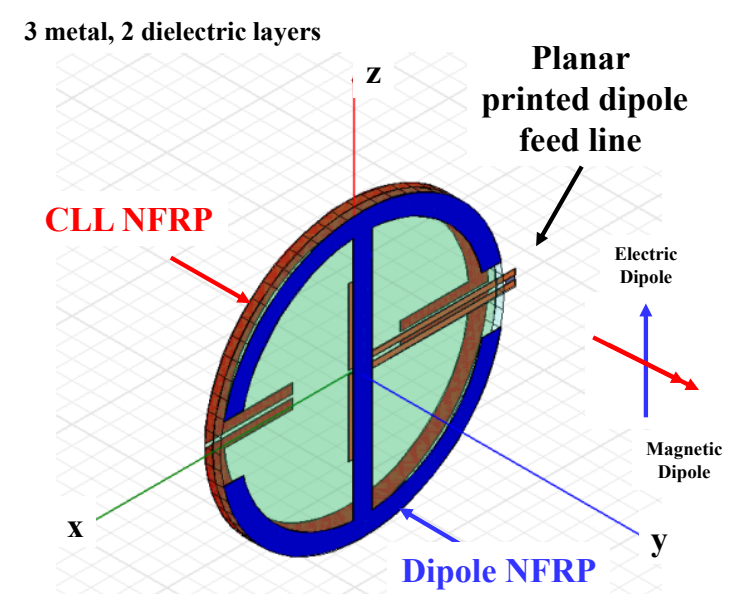

(a)

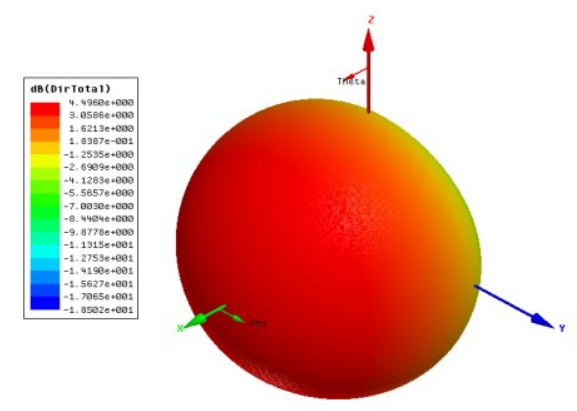

(b)

Fig. 4. Printed Huygens antenna. (a) Antenna configuration, and (b) 3D directivity pattern. Enhanced directivity occurs along the $\mathrm{x}$-axis from the presence of both electric and magnetic dipole NFRP elements. [14] 


\section{CONCLUSION}

Several combinations of metamaterials, metamaterialinspired electrically small NFRP elements, and driven electrically small radiators were reviewed. The ability to design electrically small antenna systems with combinations of these elements to achieve higher directivity than can be achieved with any single electrically small antenna will be emphasized in my presentation. I will discuss different classes of dipole-based ESAs that are combined with AMCs, are put together into end-fire array configurations, are augmented with non-Foster-based NFRP elements, and are combined together to realize Huygens sources.

Note that while the Huygens source in Fig. 4 achieves the desired functionality, it is not a broadside radiating, conformal element, i.e., the direction of maximum directivity is along the $\mathrm{x}$-direction, not along the $\mathrm{y}$ direction. An antenna that is planar and radiates into one half space orthogonal to the plane containing it would have many practical applications. Recent progress to achieve such a conformal, broadside Huygens source will be discussed in my presentation.

\section{ACKNOWLEDGMENT}

Contributions to this work occurred while the author was the 2014-2015 Australian DSTO Fulbright Distinguished Chair in Advanced Science and Technology. He especially would like to thank the Australian-American Fulbright Commission and the Defense Sciences and Technology Group (formally DSTO) for their support.

\section{REFERENCES}

[1] R. F. Harrington, "On the gain and beamwidth of directional antennas," IEEE Trans. Antennas Propag., vol. 6, no. 3, pp. 219-225, Jul. 1958.

[2] R. F. Harrington, "Effect of antenna size on gain, bandwidth and efficiency," J. Res. Nat. Bur. Stand, vol. 64-D, pp. 1-12, Jan./ Feb. 1960

[3] C. A. Balanis, Antenna Theory, $3^{\text {rd }}$ Ed., John Wiley \& Sons, Hoboken, NJ, 2005.

[4] F. Yang and Y. Rahmat-Samii, Electromagnetic Band Gap Structures in Antenna Engineering. Cambridge: UK: Cambridge University Press, 2009.

[5] P. Jin, and R. W. Ziolkowski, "High-directivity, electrically small, lowprofile near-field resonant parasitic antennas," IEEE Antennas Wirel. Propag. Lett., vol. 11, pp. 305-309, 2012.

[6] A. Erentok, P. Luljak, R. W. Ziolkowski "Antenna performance near a volumetric metamaterial realization of an artificial magnetic conductor,' IEEE Trans. Antennas Propag., vol. 53, no. 1, pp. 160-172, Jan. 2005.
[7] E. E. Altshuler, T. H. O’Donnell, A. D. Yaghjian, and S. R. Best, "A monopole superdirective array," IEEE Trans. Antennas Propag., vol. 53, no.8, pp. 2653-2661, Aug. 2005.

[8] A. D. Yaghjian, T. H. O’Donnell, E. E. Altshuler, and S. R. Best, "Electrically small supergain end-fire arrays," Radio Sci., vol.43, no. RS3002, pp. 1-13, 2008.

[9] S. Lim, and H. Ling, "Design of a closely spaced, folded Yagi antenna," IEEE Antennas Wirel. Propag. Lett., vol. 5, pp. 302-305, 2006.

[10] A. Noguchi, and H. Arai, "3-element super-directive end-fire array with decoupling network," Proceedings of ISAP 2014, Kaohsiung, Taiwan, Dec. 2-5, 2014

[11] R. W. Ziolkowski, M.-C. Tang and N. Zhu, "An efficient, broad bandwidth, high directivity, electrically small antenna," Microw. Opt. Technol. Lett., vol. 55, no. 6, pp. 1430-1434, June 2013.

[12] M.-C. Tang, R. W. Ziolkowski, S. Xiao, and M. Li, "A high-directivity, wideband, efficient, electrically small antenna system," IEEE Trans. Antennas Propag., vol. 62, no. 12, pp. 6541 - 6547, Dec. 2014.

[13] M.-C. Tang, and R. W. Ziolkowski, "Efficient, high directivity, large front-to-back-ratio, electrically small, near-field-resonant-parasitic antenna," IEEE Access, vol. 1, no. 1, pp. 16 - 28, May 2013

[14] P. Jin, and R. W. Ziolkowski, "Metamaterial-inspired, electrically small Huygens sources," IEEE Antennas Wirel. Propag. Lett., vol. 9, pp. $501-$ 505, 2010.

[15] T. Niemi, P. Alitalo, A. O. Karilainen, and S.A. Tretyakov, "Electrically small Huygens source antenna for linear polarization," IET Microw. Antennas Propag., Vol. 6, Iss. 7, pp. 735-739, 2012.

[16] R. F. Jimenez Broas, D. F. Sievenpiper and E. Yablonovitch, "A highimpedance ground plane applied to a cellphone handset geometry," IEEE Trans. Microwave Theory Tech., vol. 49, no. 7, pp. 1262-1265, July 2001.

[17] S. Clavijo, R. E. Diaz and W. E. McKinzie, "Design methodology for Sievenpiper high-impedance surfaces: An artificial magnetic conductor for positive gain electrically small antennas," IEEE Trans. Antennas Propag., vol. 51, no. 10, pp. 2678-2690, Oct. 2003.

[18] R. Coccioli, F.-R. Yang, K.-P. Ma and T. Itoh, "Aperture-coupled patch antenna on UC-PBG substrate," IEEE Trans. Microwave Theory Tech., vol. 47, no. 11, pp. 2123-2130, Nov. 1999.

[19] M.-C. Tang, B. Zhou and R. W. Ziolkowski, "Flexible uniplana electrically small directive antenna empowered by a modified CPWfeed," IEEE Antennas Wireless Propag. Lett., in press, appeared on-line Aug. 2015.

[20] R. W. Ziolkowski, P. Jin, and C.-C. Lin, "Metamaterial-inspired engineering of antennas," Proc. IEEE, vol.99, no.10,pp.1720-1731,Oct. 2011.

[21] N. Zhu and R. W. Ziolkowski, "Broad bandwidth, electrically small antenna augmented with an internal non-Foster element," IEEE Antennas Wireless Propag. Lett., vol. 11, pp. 1116-1120, 2012.

[22] N. Zhu and R. W. Ziolkowski, "Broad bandwidth, electrically small, non-Foster element-augmented antenna designs, analyses, and measurements," IEICE Transactions on Communications, vol. E96-B, no.10, pp. 2399-2409, Oct. 2013. 